\section{Braun, Fan, Haenen, Stanciu, and Theil to chair 2015 MRS Spring Meeting}

www.mrs.org/spring2015

$\mathrm{M}$ eeting chairs for the 2015 Materials Research Society (MRS) Spring Meeting are Artur Braun (Empa-Swiss Federal Laboratories for Materials Science and Technology, Switzerland), Hongyou Fan (Sandia National Laboratories, USA), Ken Haenen (Hasselt University and IMEC vzw, Belgium), Lia Stanciu (Purdue University, USA), and Jeremy A. Theil (Quantumscape, Inc., USA). The Meeting will be held April 6-10, 2015, in San Francisco, Calif.

Artur Braun is research group leader and principal investigator at EmpaSwiss Federal Laboratories for Materials Science and Technology. He is a physicist from RWTH Aachen in Germany. He holds a doctoral degree in electrochemistry from ETH Zürich.

In his early career, he worked on ultrathin magnetic films at KFA Jülich, and had brief appointments with Philips Research Laboratories on electroceramics and Mitsubishi Semiconductors Europe on dynamic random-access memory (DRAM) quality assurance. His PhD studies on supercapacitors at ETH
Zürich and the Paul Scherrer Institut led to his first MRS Meeting: the 1999 MRS Spring Meeting in San Francisco. Soon after, he moved to Lawrence Berkeley National Laboratory (LBNL) as a postdoctoral fellow researching lithium batteries and protein spectroscopy. With his expertise on carbon and synchrotron radiation, he later joined the University of Kentucky, where he worked on fossil fuel liquefaction and on carbonaceous air pollutants.

In addition to research projects in Switzerland and Europe, he is principal investigator for energy materials projects with Hawaii, India, Korea, and South Africa. He is a Marie Curie Fellow, JUSAP Board Member for the Swiss Light Source, and Advanced Light Source User Executive Committee Member at LBNL. He is Energy Materials Editor for Elsevier's Current Applied Physics and has served as an expert on renewables for the Intergovernmental Panel on Climate Change. Braun's focus is now on materials and bio-hybrids for artificial photosynthesis.
Hongyou Fan is a principal member of the technical staff at Sandia National Laboratories and a joint professor in the Department of Chemical and Biological Engineering and Center for Micro Engineered Materials at the University of New Mexico. He received a BS degree in chemistry at Jilin University, a MS degree in polymer chemistry and physics in 1995 from the Chinese Academy of Sciences, and a $\mathrm{PhD}$ degree in nanoporous materials and composites in 2000 from the University of New Mexico.

His research focuses on the development of new synthesis methods and self-assembly processes to fabricate multifunctional nanomaterials, fundamentally understanding self-assembly mechanism, structural evolution from molecular level to nano- and microscale, and structure-property relationships.

Fan's awards include the Sandia National Laboratories' Laboratory Directed Research and Development Award for Excellence in 2007, the R\&D Magazine's R\&D 100 Award in 2007 and 2010, the Federal Laboratory Consortium's Outstanding Technology Development Award in 2008 and 2013, the University of New Mexico Outstanding Faculty Mentor Award in 2005, and the Asian American Engineer of the Year Award in 2012.

Ken Haenen is a professor, vice dean of the Faculty of Sciences, and vice director of the Doctoral School for Sciences \& Technology at Hasselt
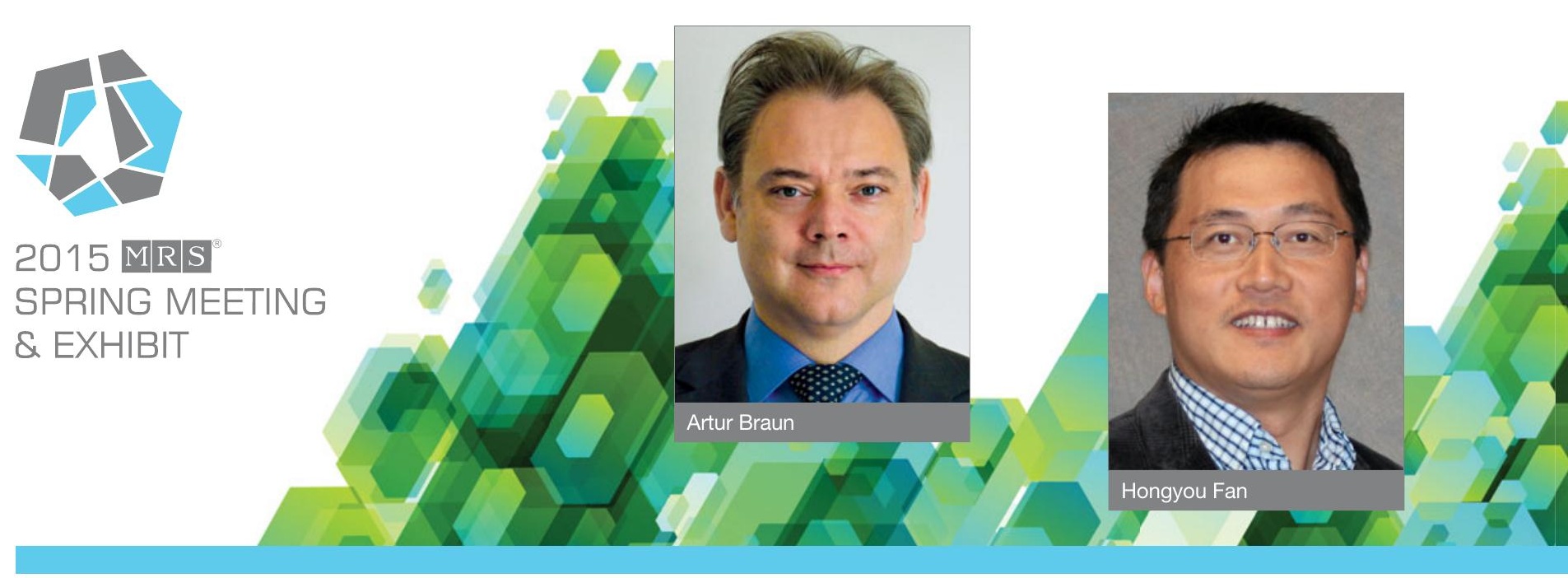
